\title{
Oxidized Avidin
}

National Cancer Institute

\section{Source}

National Cancer Institute. Oxidized Avidin. NCI Thesaurus. Code C113800.

An oxidized form of the glycoprotein avidin, that can be used as a linking agent for tissuepretarg eted radionuclide therapy. Upon intralesional administration, the aldehyde groups of oxidized avidin strongly bind to the amino groups on tissue proteins, via the formation of Schiff bases. As avidin is able to strongly bind to biotin, intravenous administration of radiolabeled biotin may lead to the selective eradication of the pre-targeted tumor cells. 